OPEN ACCESS

Edited by:

Hugo Borges Sarmento, University of Coimbra, Portugal

Reviewed by:

Pedro Gaspar

University of Coimbra, Portugal

Cynthia S. T. Wu,

Hong Kong Polytechnic University,

Hong Kong

*Correspondence:

Chunhua Zhang

zch20080808@126.com

Specialty section:

This article was submitted to

Children and Health,

a section of the journal

Frontiers in Public Health

Received: 10 May 2020

Accepted: 24 July 2020

Published: 02 September 2020

Citation:

Li Y, Xia X, Meng F and Zhang C (2020) Association Between Physical

Fitness and Anxiety in Children: A

Moderated Mediation Model of Agility

and Resilience.

Front. Public Health 8:468.

doi: 10.3389/fpubh.2020.00468

\section{Association Between Physical Fitness and Anxiety in Children: A Moderated Mediation Model of Agility and Resilience}

\author{
Yansong $\mathrm{Li}^{1}$, Xue Xia ${ }^{2}$, Fanying Meng ${ }^{3}$ and Chunhua Zhang ${ }^{1 *}$ \\ ${ }^{1}$ School of Kinesiology, Shanghai University of Sport, Shanghai, China, ${ }^{2}$ School of Psychology, Shanghai University of Sport, \\ Shanghai, China, ${ }^{3}$ Institute of Physical Education, Huzhou University, Huzhou, China
}

Background: Anxiety is one of the most prevalent mental health problems in children. Although physical fitness as a predictor of mental health, the mechanisms underlying any association between physical fitness and anxiety in children have been understudied. Thus, the aim of the present study was to determine whether an association exists between physical fitness and anxiety and to explore the roles of agility and resilience in such an association.

Methods: This cross-sectional study investigated 269 children aged 7 to 12 years from three public primary schools in Shanghai (China). Physical fitness and agility were objectively measured, and resilience and anxiety were assessed using self-reported questionnaires. The moderated mediation model was examined using the SPSS PROCESS macro, in which the moderator variable was agility, and the mediator variable was resilience.

Results: Physical fitness was inversely associated with anxiety. Resilience partially and indirectly mediated this association, and agility moderated the association between physical fitness and resilience. Physical fitness had a greater impact on resilience in children with higher agility levels.

Conclusions: Agility moderated the mediation of resilience on the indirect, inverse association between physical fitness and anxiety; thus, incorporating methods to develop agility and resilience may lead to better outcomes for physical fitness programs designed to prevent or alleviate anxiety in children.

Keywords: children, physical fitness, agility, resilience, anxiety, moderated mediation

\section{INTRODUCTION}

Anxiety is one of the most prevalent mental health problems among children $(1,2)$, with an estimated prevalence up to $20 \%(3,4)$. Anxiety in children has a negative impact on their school performance (5), social functioning (6), and quality of life (7). In addition, anxiety symptoms during childhood tend to be chronic and may lead to anxiety disorders and other serious psychopathological consequences that persist into later childhood and adulthood $(1,8,9)$. Indeed, approximately half the anxiety disorders diagnosed in adults have an onset before 11 years of 
age (10). In addition to the potential long-term effects on individuals' growth and development, children's anxiety disorders place a burden on society via direct and indirect costs (11); public expenses are more than 20 times as high for a child with vs. without clinically relevant anxiety (12). Hence, to mitigate these negative consequences of childhood anxiety, identifying the factors that affect anxiety and their underpinning mechanisms may contribute to the development of effective prevention and treatment interventions to benefit individuals as well as society.

Previous research has shown various protective factors for anxiety, such as self-efficacy (13), coping style (14), and social support (15). A positive role for physical activity in the prevention of anxiety has also been shown in several types of recent studies (16-18). Physical fitness can be defined as the capacity to perform physical activity (19), and physical fitness is more predictive of health outcomes than is physical activity in children (20). Thus, given the established health benefits of physical activity broadly, increasing evidence indicates a need to explore physical fitness. Physical fitness is considered one of the most important health markers and predictors of future risk of mental health issues $(21,22)$. In addition, physical fitness also serves as a buffer against stress and stress-related disorders (23). Anxiety is a stress-related mental health problem, but the association between physical fitness and anxiety is still unclear $(24,25)$. Therefore, investigating the mechanisms underlying the role physical fitness plays in childhood anxiety is critically needed.

To date, it is not well-understood which factors are crucial for the maintenance of physical and mental health. Resilience is a relatively new construct that may be an important factor (26). Resilience is the ability to successfully adapt to stress, trauma, or adversity, enabling individuals to avoid stress-induced mental disorders, such as depression, posttraumatic stress disorder, and anxiety $(27,28)$. In this regard, exploring how to build resilience may be useful in assisting children affected by anxiety. On the other hand, physical fitness may confer resilience owing to its stress-buffering effects (23). Thus, from a stress perspective, physical fitness, and anxiety may be linked through resilience.

In addition, agility, as the main component of physical fitness, is a combination of physical qualities and cognitive components $(22,29)$. However, unlike studies examining other main components (e.g., cardiorespiratory fitness, muscular fitness), few studies have focused on the contribution of agility to mental health (19). Within the limited literature, agility was summarized as a major input that enables resilience (30). Research has also reported that regular exposure to diverse agility-type movement challenges may facilitate the movement efficiency of athletes and their resilience to numerous dimensions of movement stress (31). Thus, whether the benefits of physical fitness on childhood anxiety may be enhanced by resilience and the role that agility plays in these relationships warrant further attention.

Therefore, the present study examined the associations among physical fitness, agility, resilience, and anxiety in children. On the basis of previous research results, we proposed the following three hypotheses: (1) physical fitness is inversely associated

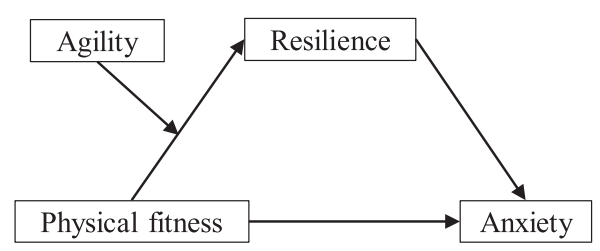

FIGURE 1 | The proposed moderated mediation model to be tested.

with anxiety in children; (2) resilience mediates the association between physical fitness and anxiety; and (3) agility moderates the indirect effect of physical fitness on anxiety through resilience, with the association becoming stronger when agility is high and weaker when agility is low. Based on these hypotheses, we proposed a theoretical model to be tested (Figure 1). In the proposed model, physical fitness plays a role in enhancing resilience and ameliorating anxiety, and the influence of physical fitness on anxiety is mediated by resilience, with the strength of this mediation conditional depending on the level of agility.

\section{MATERIALS AND METHODS}

\section{Participants}

The participants were selected from three primary schools in Shanghai (China). A sampling of schools was stratified according to physical fitness with three levels, with the first quartile being the lowest, followed by second and third quartiles, and the highest being the fourth quartile, as described and used in previous studies $(32,33)$. The current three schools were chosen for their representativeness of each level, considering the accessibility to our research team and the availability of the teachers to assist with logistics. Potential participants who had not received a diagnosis of any disease that made it impossible to complete the test and who understood each item in the questionnaires were included. Participants whose physical fitness test results exceeded the logical limits or whose questionnaires were considered invalid were excluded. After the aim of the study was explained, participants completed the objective measurements and self-reported questionnaires under the supervision of trained volunteers. Participants' names were substituted with codes in the data collection to protect privacy, and they were given an inexpensive gift as a token of appreciation. The results obtained from the tests were offered to the school administrators to provide suggestions for participants' physical activity and mental health.

Valid variables were obtained from 269 participants. The participants were in school grades 2 through 5 and comprised 126 boys and 143 girls, with ages ranging from 7-12 years (mean age $=9.75$ years; $\mathrm{SD}=1.17$ years). The number (percentage) of participants by school grade was $72(26.8 \%)$ in second grade, 67 $(24.9 \%)$ in third grade, $66(24.5 \%)$ in fourth grade, and $64(23.8 \%)$ in fifth grade.

The study was conducted in accordance with the recommendations of the World Medical Association's 
TABLE 1 | Fitness components and weights of CNSPFS scores in school-age children.

\begin{tabular}{|c|c|c|c|c|c|}
\hline \multicolumn{2}{|c|}{ Second grade } & \multicolumn{2}{|c|}{ Third and fourth grades } & \multicolumn{2}{|c|}{ Fifth grade } \\
\hline Fitness component & Weight (\%) & Fitness component & Weight (\%) & Fitness component & Weight (\%) \\
\hline BMl & 15 & $\mathrm{BMl}$ & 15 & $\mathrm{BMl}$ & 15 \\
\hline Vital capacity of lung & 15 & Vital capacity of lung & 15 & Vital capacity of lung & 15 \\
\hline 50 m sprint & 20 & 50 m sprint & 20 & $50 \mathrm{~m}$ sprint & 20 \\
\hline Sit and reach & 30 & Sit and reach & 20 & Sit and reach & 10 \\
\hline \multirow[t]{3}{*}{ Timed rope-skipping } & 20 & Timed rope-skipping & 20 & Timed rope-skipping & 10 \\
\hline & & Timed sit-ups & 10 & Timed sit-ups & 20 \\
\hline & & & & $50 \mathrm{~m} \times 8$ shuttle run & 10 \\
\hline
\end{tabular}

BMI, Body Mass Index; CNSPFS, Chinese National Student Physical Fitness Standard.

Declaration of Helsinki as revised in 1989 and was approved by the Shanghai University of Sport Ethics Committee (Shanghai, China). Written informed consent was obtained from the guardians of all participants.

\section{Measures \\ Physical Fitness}

To minimize variability, all physical fitness tests were conducted by trained volunteers from the Shanghai Research Center for Physical Fitness and Health of Children and Adolescents. The volunteers all majored in kinesiology and were already familiar with the testing methods.

Physical fitness was measured using the Chinese National Student Physical Fitness Standard (CNSPFS), a standardized test commonly used in Chinese schools (34). As indicated in the CNSPFS guidelines, the results of different fitness components were scored according to sex and school grade level. The total physical fitness score was composed of the product sum of the scores and weights of each component. The weights of the CNSPFS scores for each fitness component stratified by school grade are given in Table $\mathbf{1}$.

\section{Agility}

Agility was assessed using the side-step test, which is an effective and commonly used measure (35). For this test, the participant stood over a central line. At the start of the test, the participant moved laterally, side-stepping toward a far-right line $(1 \mathrm{~m}$ from the central line) until the right foot crossed the far-right line. Once the right foot crossed that line, the participant changed directions and moved laterally, side-stepping to the left until the left foot crossed the far-left line ( $1 \mathrm{~m}$ from the central line). After reaching the far-left line, the participant moved laterally to return to the central line. This motion was repeated for $20 \mathrm{~s}$, and one point was given for each line passed. The test was conducted twice, and the highest side-step score was recorded.

\section{Resilience}

Resilience was obtained using the Resilience Scale for Chinese Adolescents (RSCA), which has shown excellent psychometric properties and has been widely used in evaluating the resilience of Chinese children (36). It includes 27 items that have been classified into five factors: target concentration, emotional control, positive thinking, family support, and interpersonal assistance. Participants were asked to rate themselves on questions using a 5-point Likert scale ranging from a score of 1 , indicating "not true at all," to 5, indicating "true nearly all the time." The total score for the RSCA ranged from 27 to 135, with a higher score indicating a higher level of resilience. Cronbach's alpha for this test in the present study was 0.74 .

\section{Anxiety}

Anxiety was defined using the Chinese version of the Multidimensional Anxiety Scale for Children (MASC), a 39-item self-report scale for assessing children's anxiety (37). The items clustered into the following four scales: physical symptoms, harm avoidance, social anxiety, and separation anxiety. Response options ranged from 0 , indicating "never true," to 3 , indicating "often true." The total score (range, 0-117) was generated by adding the scores of all items; thus, a higher score reflected a greater degree of anxiety. Cronbach's alpha for this scale in this study was 0.89 .

\section{Statistical Analysis}

The data were analyzed using SPSS, version 22.0, and the PROCESS macro program for SPSS (38). Both graphical (normal probability plots) and statistical (Kolmogorov-Smirnov test) methods were used to examine the nature of the variables, and all were found to fit a normal distribution. Thus, parametric statistics were used. Harman's single-factor test was conducted, and the results indicated that no serious method bias existed in the present study.

We conducted descriptive statistics for the main study variables (reported as means \pm standard deviations) and Pearson correlation for bivariate associations (reported as values of $r$ ). We then used the PROCESS macro to perform a regressionbased path analysis, which is similar to structural equation modeling but takes into consideration irregular sampling distributions (39). As noted by Edwards and Lambert (40), the distribution of the indirect effect can be non-normal even if the constituent variables are normal. Given this possibility, all regression coefficients were tested using the bias-corrected percentile Bootstrap method $(41,42)$. We tested the theoretical hypothesis model, controlled for age and sex, by estimating the $95 \%$ confidence intervals (CIs) for the mediation and 
TABLE 2 | Descriptive statistics and correlations between variables.

\begin{tabular}{|c|c|c|c|c|c|}
\hline Variable & Mean \pm SD & 1 & 2 & 3 & 4 \\
\hline (1). Physical fitness & $81.50 \pm 9.80$ & - & & & \\
\hline (2). Agility & $37.18 \pm 9.03$ & $0.408^{\star \star \star}$ & - & & \\
\hline (3). Resilience & $93.10 \pm 13.77$ & $0.248^{\star \star \star}$ & $0.267^{\star \star \star}$ & - & \\
\hline (4). Anxiety & $43.33 \pm 19.18$ & $-0.244^{\star \star \star}$ & $-0.154^{*}$ & $-0.247^{\star \star \star}$ & - \\
\hline
\end{tabular}

$S D$, standard deviation; ${ }^{*} p<0.05,{ }^{\star * *} p<0.001$.

TABLE 3 | Mediation modeling results assessing the effect of physical fitness on anxiety.

\begin{tabular}{|c|c|c|c|c|c|c|}
\hline Effect & Path & $\beta$ & SE & $t$ & LLCI & ULCI \\
\hline Total effect & Physical fitness-anxiety & -0.256 & 0.060 & $-4.261^{\star \star \star}$ & -0.374 & -0.137 \\
\hline \multirow[t]{3}{*}{ Direct effect } & Physical fitness-resilience & 0.249 & 0.060 & $4.139^{\star \star \star}$ & 0.131 & 0.367 \\
\hline & Resilience-anxiety & -0.190 & 0.060 & $-3.145^{\star \star}$ & -0.308 & -0.071 \\
\hline & Physical fitness-anxiety & -0.208 & 0.061 & $-3.423^{\star \star}$ & -0.328 & -0.089 \\
\hline Indirect effect & Physical fitness-resilience-anxiety & -0.047 & 0.019 & - & -0.088 & -0.013 \\
\hline
\end{tabular}

$\beta$, standardized coefficients; SE, standard error; LLCI and ULCI, lower level and upper level of the bias-corrected $95 \%$ bootstrap confidence interval; ** $p<0.01,{ }^{* \star \star} p<0.001$.

moderation effects, with 5,000 resampled samples. When a $95 \%$ CI did not include 0 , the result was considered statistically significant. We selected the Model 4 in PROCESS to examine the simple mediation effect of resilience on the association between physical fitness and anxiety. We then incorporated the proposed moderator variable (agility) into the model by conducting moderated mediation (also known as a conditional indirect effect) analysis using the Model 7 in PROCESS to determine whether the indirect path was conditionally moderated by the "strength" or value of agility. This model uses ordinary least squares regression to assess the conditional indirect effect and tests the effect with bootstrap CIs for different values of the moderating variable (agility) to determine whether the indirect effect varies. Before formal data analysis, all variables were standardized. Values of $p<0.05$ were considered statistically significant.

\section{RESULTS}

Descriptive statistics and correlations of the study variables are shown in Table 2. We found that physical fitness, agility, and resilience were inversely correlated with anxiety; physical fitness was directly correlated with agility and resilience; and agility was directly correlated with resilience.

As shown in Table 3, the results of simple mediation model testing using Model 4 indicated that physical fitness was directly associated with resilience $(\beta=0.249, t=4.139, p<0.001)$. Resilience was inversely associated with anxiety $(\beta=-0.190$, $t=-3.145, p<0.01)$. The association between physical fitness and anxiety was also significant $(\beta=-0.208, t=-3.423, p<$ 0.01 ). We also found a significant indirect effect of physical fitness on anxiety via resilience: the bootstrapping results indicated an indirect effect $[\beta=-0.047 ; 95 \% \mathrm{CI}:(-0.088,-0.013)]$. The indirect effect accounted for $18.4 \%$ of the total effect, suggesting that resilience played a partial mediating role in the association between physical fitness and anxiety. These findings supported our first and second study hypotheses.

Moderated mediation analysis using Model 7 was conducted to assess whether anxiety was indirectly affected by physical fitness via mediation through resilience and whether this effect was conditionally moderated by agility. We hypothesized that children with higher agility scores would show a stronger association between physical fitness and resilience compared with those with lower agility scores. Table 4 shows the results of the moderated mediation model test. The effect of the interaction between physical fitness and agility on resilience was statistically significant $(\beta=0.167, t=2.597, p<0.05)$. Figure 2 illustrates the interaction at high (plus 1 SD) and low (minus 1 SD) levels of physical fitness and agility. The plots indicate the interaction between physical fitness and agility on resilience and suggested that for children with higher agility levels, there was a stronger positive association between physical fitness and resilience compared with children having lower agility levels.

The conditional indirect effect of physical fitness on anxiety through resilience at various values of agility was analyzed when the agility score was the sample mean and also at plus or minus 1 $\mathrm{SD}$. The results revealed that the conditional indirect effect was significant at the mean and at a high level (plus 1 SD) of the moderator (agility), but not at a low level (minus $1 \mathrm{SD}$ ), and the bootstrap 95\% CIs supported these results (see Table 5). In addition, the moderated mediation index was also statistically significant. These results supported our third study hypothesis because the association between physical fitness and the outcome variables became more evident as the moderator value increased.

\section{DISCUSSION}

In the present study, we used a moderated mediation model analysis to assist in understanding the mechanisms underlying any association between physical fitness and anxiety in children. Our results supported our three study hypotheses, namely, that 
TABLE 4 | Moderated mediation modeling results assessing the effect of physical fitness on anxiety.

\begin{tabular}{|c|c|c|c|c|c|c|c|c|}
\hline Outcome variable & Factor & $\beta$ & SE & $t$ & LLCI & ULCI & $R^{2}$ & $\boldsymbol{F}$ \\
\hline \multirow[t]{5}{*}{ Resilience } & Physical fitness & 0.222 & 0.067 & $3.312^{\star \star}$ & 0.090 & 0.353 & 0.119 & $7.129^{\star \star \star}$ \\
\hline & Agility & 0.144 & 0.068 & $2.119^{\star}$ & 0.010 & 0.278 & & \\
\hline & Physical fitness $\times$ agility & 0.167 & 0.064 & $2.597^{\star}$ & 0.040 & 0.293 & & \\
\hline & Age & 0.045 & 0.052 & 0.859 & -0.058 & 0.147 & & \\
\hline & Sex & -0.052 & 0.118 & -0.444 & -0.283 & 0.179 & & \\
\hline \multirow[t]{4}{*}{ Anxiety } & Physical fitness & -0.208 & 0.061 & $-3.423^{\star \star}$ & -0.328 & -0.089 & 0.109 & $8.061^{\star \star \star}$ \\
\hline & Resilience & -0.190 & 0.060 & $-3.145^{\star \star}$ & -0.308 & -0.071 & & \\
\hline & Age & -0.052 & 0.050 & -1.031 & -0.150 & 0.047 & & \\
\hline & Sex & 0.187 & 0.118 & 1.586 & -0.045 & 0.419 & & \\
\hline
\end{tabular}

$\beta$, standardized coefficients; SE, standard error; LLCl and ULCI, lower level and upper level of the bias-corrected $95 \%$ bootstrap confidence interval; ${ }^{\star} p<0.05,{ }^{\star *} p<0.01,{ }^{* \star *} p<0.001$.

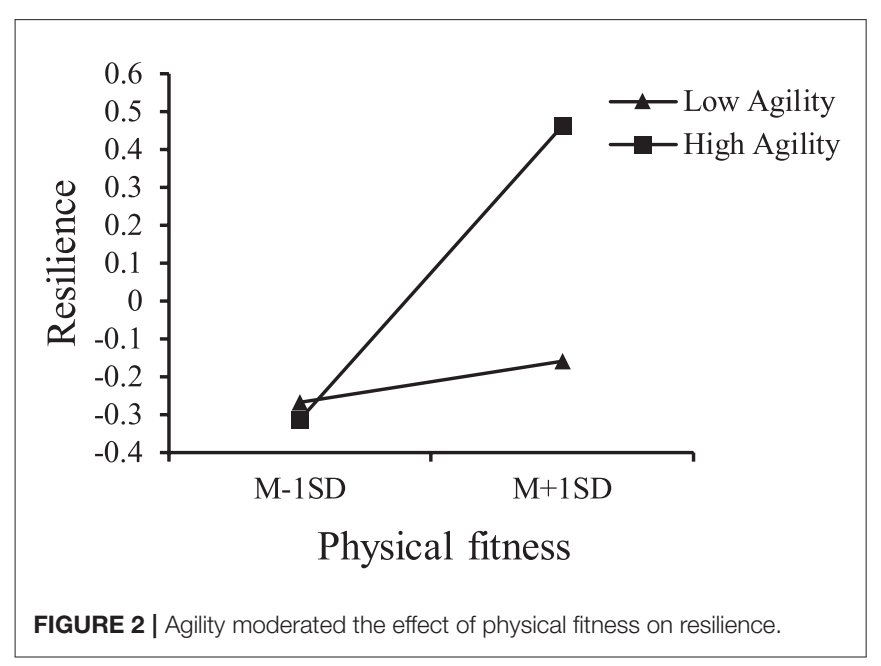

TABLE 5 | Moderated mediation effect of physical fitness on anxiety at specific conditional values of agility.

\begin{tabular}{lcccc}
\hline & \multicolumn{5}{c}{ Specific conditional values of agility } \\
\cline { 2 - 5 } & $\beta$ & SE & LLCI & ULCI \\
\hline-1 SD & -0.010 & 0.015 & -0.046 & 0.017 \\
Mean & -0.042 & 0.017 & -0.077 & -0.010 \\
+1 SD & -0.074 & 0.030 & -0.134 & -0.017 \\
\hline & & Index of moderated mediation & \\
\hline Agility & Index & SE & LLCI & ULCI \\
\hline
\end{tabular}

$\beta$, standardized coefficients; SD, standard deviation; SE, standard error; LLCI and ULCI, lower level and upper level of the bias-corrected 95\% bootstrap confidence interval.

(1) higher physical fitness levels were associated with lower anxiety levels in children, (2) resilience mediated physical fitness and anxiety, and (3) agility moderated the mediation of resilience on the association, which was stronger in children with higher levels of agility.

Our results suggested that higher levels of physical fitness were associated with lower anxiety levels in children. A plausible explanation for this finding is that individuals who display higher levels of physical fitness tend to have more positive perceptions of anxiety symptoms and thus feel less anxious (25). This supposition is supported by several studies that have shown that improvements in physical fitness are associated with reductions in levels of anxiety (43-45). However, RodriguezAyllon et al. (19) found no relationship between physical fitness components and anxiety indicators. In addition to the demographic characteristics of the participants, these discrepant results may be because those previous studies focused on specific components of physical fitness (46), but physical fitness components relate in different ways to the various aspects of mental health (19). Our study results were based on the total physical fitness scores of children obtained by CNSPFS. This comprehensive outcome reflects the basic physical fitness of children and is an important basis for judging the overall level of physical fitness in children. Further analysis of physical fitness as a protective factor may provide a new approach for the prevention or intervention of childhood anxiety. Moreover, the fitness components, standards for evaluation, and score weights vary in the CNSPFS to adjust for the growth and development of children. Thus, incorporating interventions to reduce the level of anxiety through physical fitness programs that are appropriate at each age in children may lead to better outcomes.

Our mediation model revealed that resilience mediated the association between physical fitness and anxiety, indicating that establishing good physical fitness was conducive to resilience in children, which in turn ameliorates their anxiety. Physical fitness appears to confer resilience by blunting or optimizing neuroendocrine and physiological responses (e.g., the hypothalamic-pituitary-adrenal axis, the sympathetic nervous system) to physical and psychosocial stressors (23). In line with previous studies (47-49), our result is meaningful in that it supports physical fitness being an effective manner of building resilience in children. On the other hand, resilience can also indirectly reduce the negative effects of stress on anxiety through its mediation effect $(50,51)$. As a dynamic process, resilience is construed as an active adaptation mechanism that may help alleviate anxiety (27), which was observed in our study. In other words, resilience accounting for an important part of 
the variations in anxiety symptoms suggests that interventions should target ways to enhance resilience (50).

We speculate that a biological mechanism that might provide an explanation for our findings is that physical fitness may promote resilience by minimizing inflammation. The benefits of physical fitness may, in part, be attributed to anti-inflammatory effects via changes in body composition and skeletal muscle (23). On the other hand, Interleukin- 8 as an inflammatory marker, has been suggested to be involved in the biological mechanisms mediating resilience to anxiety (52). Overall, resilience may serve as a "bridge" linking physical fitness and anxiety; thus, it would be insufficient to attempt to reduce anxiety in children by promoting physical fitness alone because resilience also plays an important role.

Our moderated mediation model offered a more detailed picture of the mechanisms that associated physical fitness with anxiety, providing a basis for improving anxiety in children using physical fitness via full consideration of the characteristics of agility. However, in research investigating the relationship between physical fitness and mental health, to the best of our knowledge, there is no in-depth analysis examining the importance of agility. Therefore, in our study, we used agility as an independent moderator to assess its influence. Interestingly, we found that physical fitness did not have a significant effect on resilience when the level of agility was low, but the positive effect of physical fitness occurred with the improvement of agility. More importantly, these results were confirmed in our physical fitness-anxiety model. These findings support our hypothesis that in relation to mental health, agility plays a critical role in the effects of physical fitness.

The self-efficacy based model of resilience suggests that executive function provides much of the capacity for resilience in individuals, with executive function enhancing self-efficacy to enable successful adjustment $(53,54)$. Additionally, executive function yields a greater propensity for resilience in children (55, 56). On the other hand, recent evidence suggests that agility is a key component for executive function, and children with higher levels of agility have shown better performance in executive function $(46,57)$. Taken together, these findings indicate that executive function combines agility with resilience-which to some extent reflects the adaptation and coping ability of children (56) - to help explain the pathway between agility and resilience and provide a new perspective for further understanding how physical fitness affects resilience. More interestingly, the sidestep test used in the present study is also considered useful for improving cardiorespiratory and muscular fitness (35). These main fitness components may all contribute to the promotion of resilience $(19,23)$. Therefore, the potential connections appear to demonstrate the value of agility, which should be taken into account for physical fitness to benefit resilience. To gain greater insight into the moderation effect of agility on the association between physical fitness and anxiety, both theoretical and empirical research studies are needed in the future.

Our study has some limitations that should be considered when interpreting our results. First, this study used a cross-sectional design, which prevented us from making any cause-and-effect conclusions. Therefore, longitudinal studies should be designed to validate the findings. Second, our study relied in part on self-reported data, which may affect the outcomes. Future studies should measure mental health objectively, such as through neuropsychological tests. Third, our findings were obtained from children in public primary schools, and whether the results are applicable to other populations will require further testing.

\section{CONCLUSIONS}

The current study offers some insight into the mechanisms underlying the association between physical fitness and anxiety. Physical fitness is a valuable factor, providing some protection against anxiety for children. This protective effect is partially mediated through the resilience, which is moderated by agility. The moderation effect may enable children with higher agility levels to show better outcomes in the association between physical fitness and anxiety. The analysis conducted in the present study using a moderated mediation model analysis expands on the current knowledge by providing evidence to support potential mechanisms. The results of this analysis suggest that incorporating methods to develop agility and to improve resilience may lead to better outcomes when designing physical fitness programs to prevent or alleviate anxiety in children.

\section{DATA AVAILABILITY STATEMENT}

The raw data supporting the conclusions of this article will be made available by the authors, without undue reservation.

\section{ETHICS STATEMENT}

The studies involving human participants were reviewed and approved by Shanghai University of Sport Ethics Committee. Written informed consent to participate in this study was provided by the participants' legal guardian/next of kin.

\section{AUTHOR CONTRIBUTIONS}

$\mathrm{YL}, \mathrm{FM}$, and $\mathrm{CZ}$ contributed to the conception and design of the study. XX and FM organized the database. YL and XX performed the statistical analysis and wrote the first draft of the manuscript. CZ revised the manuscript. All authors contributed to manuscript revision, read, and approved the submitted version.

\section{FUNDING}

This study was supported by the Shanghai Youth Sports Training Association of China.

\section{ACKNOWLEDGMENTS}

The authors thank all the participants, volunteers, teachers, and schools for their enthusiastic participation in this study. 


\section{REFERENCES}

1. Cartwright-Hatton S, McNicol K, Doubleday E. Anxiety in a neglected population: prevalence of anxiety disorders in pre-adolescent children. Clin Psychol Rev. (2006) 26:817-33. doi: 10.1016/j.cpr.2005.12.002

2. Lichtwarck-Aschoff A, van Rooij M. Are changes in children's communication patterns predictive of treatment outcomes for children with anxiety? Clin Psychol Psychother. (2019) 26:572-85. doi: 10.1002/cpp.2383

3. Chavira DA, Stein MB, Bailey K, Stein MT. Child anxiety in primary care: prevalent but untreated. Depress Anxiety. (2004) 20:155-64. doi: 10.1002/da.20039

4. Kendall PC, Safford S, Flannery-Schroeder E, Webb A. Child anxiety treatment: outcomes in adolescence and impact on substance use and depression at 7.4-year follow-up. J Consult Clin Psychol. (2004) 72:276-87. doi: 10.1037/0022-006X.72.2.276

5. Mazzone L, Ducci F, Scoto MC, Passaniti E, D’Arrigo VG, Vitiello B. The role of anxiety symptoms in school performance in a community sample of children and adolescents. BMC Public Health. (2007) 7:347. doi: 10.1186/1471-2458-7-347

6. Kertz SJ, Sylvester C, Tillman R, Luby JL. Latent class profiles of anxiety symptom trajectories from preschool through school age. J Clin Child Adolesc Psychol. (2019) 48:316-31. doi: 10.1080/15374416.2017.1295380

7. Kucian K, McCaskey U, O'Gorman Tuura R, von Aster M. Neurostructural correlate of math anxiety in the brain of children. Transl Psychiatry. (2018) 8:273. doi: 10.1038/s41398-018-0320-6

8. Bittner A, Egger HL, Erkanli A, Jane Costello E, Foley DL, Angold A. What do childhood anxiety disorders predict? J Child Psychol Psychiatry. (2007) 48:1174-83. doi: 10.1111/j.1469-7610.2007.01812.x

9. Green SA, Berkovits LD, Baker BL. Symptoms and development of anxiety in children with or without intellectual disability. J Clin Child Adolesc Psychol. (2015) 44:137-44. doi: 10.1080/15374416.2013.873979

10. Kessler RC, Berglund D, Demler O, Jin R, Merikangas KR. Lifetime prevalence and age-of-onset distributions of DSM-IV disorders in the national comorbidity survey replication. Arch Gen Psychiatry. (2005) 62:593-602. doi: 10.1001/archpsyc.62.6.593

11. Hoffman DL, Dukes EM, Wittchen HU. Human and economic burden of generalized anxiety disorder. Depress Anxiety. (2008) 25:72-90. doi: 10.1002/da.20257

12. Bodden DH, Dirksen CD, Bogels SM. Societal burden of clinically anxious youth referred for treatment: a cost-of-illness study. J Abnorm Child Psychol. (2008) 36:487-97. doi: 10.1007/s10802-007-9194-4

13. Sullivan TP, McPartland T, Price C, Cruza-Guet MC, Swan SC. Relationship self-efficacy protects against mental health problems among women in bidirectionally aggressive intimate relationships with men. J Couns Psychol. (2013) 60:641-7. doi: 10.1037/a0033406

14. Xiong W, Liu H, Gong P, Wang Q, Ren Z, He M, et al. Relationships of coping styles and sleep quality with anxiety symptoms among Chinese adolescents: a cross-sectional study. J Affect Disord. (2019) 257:108-15. doi: 10.1016/j.jad.2019.07.032

15. Hopkins PD, Shook NJ. A review of sociocultural factors that may underlie differences in African American and European American anxiety. J Anxiety Disord. (2017) 49:104-13. doi: 10.1016/j.janxdis.2017.04.003

16. Knappe F, Colledge F, Gerber M. Impact of an 8-week exercise and sport intervention on post-traumatic stress disorder symptoms, mental health, and physical fitness among male refugees living in a greek refugee camp. Int $J$ Environ Res Public Health. (2019) 16:3904. doi: 10.3390/ijerph16203904

17. Schuch FB, Stubbs B, Meyer J, Heissel A, Zech P, Vancampfort D, et al. Physical activity protects from incident anxiety: a meta-analysis of prospective cohort studies. Depress Anxiety. (2019) 36:846-58. doi: 10.1002/da.22915

18. Yan W, Wang X, Kuang H, Chen Y, Baktash MB, Eskenazi B, et al. Physical activity and blood pressure during pregnancy: mediation by anxiety symptoms. J Affect Disord. (2020) 264:376-82. doi: 10.1016/j.jad.2019.11.056

19. Rodriguez-Ayllon M, Cadenas-Sanchez C, Esteban-Cornejo I, Migueles JH, Mora-Gonzalez J, Henriksson P, et al. Physical fitness and psychological health in overweight/obese children: a cross-sectional study from the activebrains project. J Sci Med Sport. (2018) 21:179-84. doi: 10.1016/j.jsams.2017.09.019

20. Bovet P, Auguste R, Burdette H. Strong inverse association between physical fitness and overweight in adolescents: a large school-based survey. Int J Behav Nutr Phys Act. (2007) 4:24. doi: 10.1186/1479-58 $68-4-24$

21. Ramos-Sepulveda JA, Ramirez-Velez R, Correa-Bautista JE, Izquierdo M, Garcia-Hermoso A. Physical fitness and anthropometric normative values among Colombian-Indian schoolchildren. BMC Public Health. (2016) 16:962. doi: 10.1186/s12889-016-3652-2

22. Ortega FB, Ruiz JR, Castillo MJ, Sjostrom M. Physical fitness in childhood and adolescence: a powerful marker of health. Int J Obes. (2008) 32:1-11. doi: $10.1038 /$ sj.ijo.0803774

23. Silverman MN, Deuster PA. Biological mechanisms underlying the role of physical fitness in health and resilience. Interface Focus. (2014) 4:20140040. doi: 10.1098/rsfs.2014.0040

24. Kandola A, Vancampfort D, Herring M, Rebar A, Hallgren M, Firth J, et al. Moving to beat anxiety: epidemiology and therapeutic issues with physical activity for anxiety. Curr Psychiatry Rep. (2018) 20:63. doi: 10.1007/s11920-018-0923-x

25. Williams SE, Carroll D, Veldhuijzen van Zanten JJ, Ginty AT. Anxiety symptom interpretation: a potential mechanism explaining the cardiorespiratory fitness-anxiety relationship. J Affect Disord. (2016) 193:151-6. doi: 10.1016/j.jad.2015.12.051

26. Obbarius N, Fischer F, Obbarius A, Nolte S, Liegl G, Rose M. A 67-item stress resilience item bank showing high content validity was developed in a psychosomatic sample. J Clin Epidemiol. (2018) 100:1-12. doi: 10.1016/j.jclinepi.2018.04.004

27. Kalisch R, Muller MB, Tuscher O. A conceptual framework for the neurobiological study of resilience. Behav Brain Sci. (2015) 38:e92. doi: 10.1017/S0140525X15000023

28. Liu H, Zhang C, Ji Y, Yang L. Biological and psychological perspectives of resilience: is it possible to improve stress resistance? Front Hum Neurosci. (2018) 12:326. doi: 10.3389/fnhum.2018.00326

29. Sheppard JM, Young WB. Agility literature review: classifications, training and testing. J Sports Sci. (2006) 24:919-32. doi: 10.1080/02640410500 457109

30. Bowers C, Kreutzer C, Cannon-Bowers J, Lamb J. Team resilience as a secondorder emergent state: a theoretical model and research directions. Front Psychol. (2017) 8:1360. doi: 10.3389/fpsyg.2017.01360

31. Liefeith A, Kiely J, Collins D, Richards J. Back to the future in support of a renewed emphasis on generic agility training within sports-specific developmental pathways. J Sports Sci. (2018) 36:2250-5. doi: 10.1080/02640414.2018.1449088

32. Diez-Fernandez A, Martinez-Vizcaino V, Torres-Costoso A, Canete Garcia-Prieto J, Franquelo-Morales P, Sanchez-Lopez M. Strength and cardiometabolic risk in young adults: the mediator role of aerobic fitness and waist circumference. Scand J Med Sci Sports. (2018) 28:1801-7. doi: $10.1111 /$ sms. 13077

33. Garcia-Hermoso A, Esteban-Cornejo I, Olloquequi J, Ramirez-Velez R. Cardiorespiratory fitness and muscular strength as mediators of the influence of fatness on academic achievement. J Pediatr. (2017) 187:127-33 e3. doi: 10.1016/j.jpeds.2017.04.037

34. Zhu Z, Yang Y, Kong Z, Zhang Y, Zhuang J. Prevalence of physical fitness in Chinese school-aged children: findings from the 2016 physical activity and fitness in china-the youth study. J Sport Health Sci. (2017) 6:395-403. doi: 10.1016/j.jshs.2017.09.003

35. Thiebaud RS, Abe T, Bravo JC, Giovannitti N, Sullivan AP. Muscle activation and heart rate responses to a side-step interval exercise. Clin Physiol Funct Imaging. (2018) 38:285-90. doi: 10.1111/cpf.12413

36. Hu YQ, Gan YQ. Development and psychometric validity of the resilience scale for Chinese adolescents. Acta Psychologica Sinica. (2008) 40:902-12. doi: 10.3724/SP.J.1041.2008.00902

37. March JS, Parker JD, Sullivan K, Stallings P, Conners CK. The multidimensional anxiety scale for children (MASC): factor structure, reliability, and validity. J Am Acad Child Adolesc Psychiatry. (1997) 36:554-65. doi: 10.1097/00004583-199704000-00019

38. Hayes AF. Introduction to Mediation, Moderation, and Conditional Process Analysis: A Regression-based Approach. New York: The Guilford Press (2013).

39. Hayes AF, Montoya AK, Rockwood NJ. The analysis of mechanisms and their contingencies: PROCESS versus structural equation modeling. Australas Mark J. (2017) 25:76-81. doi: 10.1016/j.ausmj.2017.02.001 
40. Edwards JR, Lambert LS. Material for methods for integrating moderation and mediation: a general analytical framework using moderated path analysis. Psychol Methods. (2007) 12:1-22. doi: 10.1037/1082-989X.12.1.1

41. MacKinnon DP, Lockwood CM, Hoffman JM, West SG, Sheets V. A comparison of methods to test mediation and other intervening variable effects. Psychol Methods. (2002) 7:83-104. doi: 10.1037/1082-989X.7.1.83

42. Zhou J, Yang Y, Qiu X, Yang X, Pan H, Ban B, et al. Serial multiple mediation of organizational commitment and job burnout in the relationship between psychological capital and anxiety in Chinese female nurses: a cross-sectional questionnaire survey. Int J Nurs Stud. (2018) 83:75-82. doi: 10.1016/j.ijnurstu.2018.03.016

43. Cordoba-Torrecilla S, Aparicio VA, Soriano-Maldonado A, Estevez-Lopez F, Segura-Jimenez V, Alvarez-Gallardo I, et al. Physical fitness is associated with anxiety levels in women with fibromyalgia: the al-Andalus project. Qual Life Res. (2016) 25:1053-8. doi: 10.1007/s11136-015-1128-y

44. Gordon BR, McDowell CP, Lyons M, Herring MP. Associations between grip strength and generalized anxiety disorder in older adults: results from the irish longitudinal study on ageing. J Affect Disord. (2019) 255:136-41. doi: 10.1016/j.jad.2019.05.043

45. Zotcheva E, Pintzka CWS, Salvesen O, Selbaek G, Haberg AK, Ernstsen L. Associations of changes in cardiorespiratory fitness and symptoms of anxiety and depression with brain volumes: the HUNT study. Front Behav Neurosci. (2019) 13:53. doi: 10.3389/fnbeh.2019.00053

46. Mora-Gonzalez J, Esteban-Cornejo I, Cadenas-Sanchez C, Migueles JH, Molina-Garcia P, Rodriguez-Ayllon M, et al. Physical fitness, physical activity, and the executive function in children with overweight and obesity. J Pediatr. (2019) 208:50-6. doi: 10.1016/j.jpeds.2018.12.028

47. Chen R, Fall K, Czene K, Kennedy B, Valdimarsdottir U, Fang F. Impact of parental cancer on IQ, stress resilience, and physical fitness in young men. Clin Epidemiol. (2018) 10:593-604. doi: 10.2147/CLEP.S152210

48. Hansen AM, Gullander M, Hogh A, Persson R, Kolstad HA, Willert MV, et al. Workplace bullying, sleep problems and leisure-time physical activity: a prospective cohort study. Scand J Work Environ Health. (2016) 42:26-33. doi: 10.5271/sjweh.3537

49. Jefferies P, Ungar M, Aubertin P, Kriellaars D. Physical literacy and resilience in children and youth. Front Public Health. (2019) 7:346. doi: 10.3389/fpubh.2019.00346
50. Anyan F, Hjemdal O. Adolescent stress and symptoms of anxiety and depression: resilience explains and differentiates the relationships. J Affect Disord. (2016) 203:213-20. doi: 10.1016/j.jad.2016.05.031

51. Ma X, Wang Y, Hu H, Tao XG, Zhang Y, Shi H. The impact of resilience on prenatal anxiety and depression among pregnant women in Shanghai. J Affect Disord. (2019) 250:57-64. doi: 10.1016/j.jad.2019.02.058

52. Janelidze S, Suchankova P, Ekman A, Erhardt S, Sellgren C, Samuelsson $\mathrm{M}$, et al. Low IL-8 is associated with anxiety in suicidal patients: genetic variation and decreased protein levels. Acta Psychiatr Scand. (2015) 131:26978. doi: 10.1111/acps.12339

53. Masten AS, Tellegen A. Resilience in developmental psychopathology: contributions of the project competence longitudinal study. Dev Psychopathol. (2012) 24:345-61. doi: 10.1017/S095457941200003X

54. Zhang Y, Zhang X, Zhang L, Guo C. Executive function and resilience as mediators of adolescents' perceived stressful life events and school adjustment. Front Psychol. (2019) 10:446. doi: 10.3389/fpsyg.2019.00446

55. Greenberg MT. Promoting resilience in children and youth: preventive interventions and their interface with neuroscience. Ann N Y Acad Sci. (2006) 1094:139-50. doi: 10.1196/annals.1376.013

56. Obradović J. Physiological responsivity and executive functioning: implications for adaptation and resilience in early childhood. Child Dev Perspect. (2016) 10:65-70. doi: 10.1111/cdep.12164

57. Mora-Gonzalez J, Esteban-Cornejo I, Cadenas-Sanchez C, Migueles JH, Rodriguez-Ayllon M, Molina-Garcia P, et al. Fitness, physical activity, working memory, and neuroelectric activity in children with overweight/obesity. Scand J Med Sci Sports. (2019) 29:1352-63. doi: 10.1111/sms.13456

Conflict of Interest: The authors declare that the research was conducted in the absence of any commercial or financial relationships that could be construed as a potential conflict of interest.

Copyright $(2020 \mathrm{Li}, \mathrm{Xia}$, Meng and Zhang. This is an open-access article distributed under the terms of the Creative Commons Attribution License (CC BY). The use, distribution or reproduction in other forums is permitted, provided the original author(s) and the copyright owner(s) are credited and that the original publication in this journal is cited, in accordance with accepted academic practice. No use, distribution or reproduction is permitted which does not comply with these terms. 